\title{
Simulation of the rock caving process using a mesh-free method
}

\author{
S. Karekal CSIRO Earth Science and Resource Engineering, Australia \\ R. Das CSIRO Mathematical and Information Sciences, Australia
}

L. Mosse Leap Australia, Australia

P.W. Cleary CSIRO Mathematical and Information Sciences, Australia

\begin{abstract}
Understanding the caving process and material flow is fundamental to block and sublevel cave mining, and also longwall mining in sedimentary deposits. However, due to complexity of the structure of the rock mass and its behaviour, the caving process is rarely understood and cannot be described by simple mathematical models. Numerical methods that solve the governing partial differential equations with appropriate boundary conditions have been employed in modelling geomechanics problems. Notably, mesh-based finite element, boundary element and finite difference methods or combinations of these methods have been employed effectively for stress analysis for simulating rock excavations. However, when modelling caving processes, such as block caving, sublevel caving and longwall caving, the traditional mesh-based continuum approaches may fail to simulate such a process due to large material movement, fracture and fragmentation. This is mainly due to inaccuracies arising from mesh distortions resulting in spurious and ill conditioned models, and their deficiency to capture large material flows and fragmentations. In this paper, an application of a mesh-free method, called smoothed particle hydrodynamics (SPH), is demonstrated for simulating rock caving in a stratified deposit. The advantages for simulating caving processes using a mesh-free numerical framework are highlighted. Two different examples are chosen, one with elastic-brittle material behaviour with a large yield strength (failure by brittle fracture) and the other with elasto-plastic material behaviour with a relatively lower yield strength to allow ductile deformation of the rock mass. The modelling results capture the mechanistic aspects observed in caving processes. It is shown that the largescale deformation and the associated fracture processes in caving can be effectively simulated using SPH. In the authors' knowledge, this work is the first attempt in demonstrating the application of a mesh-free method for simulating caving processes.
\end{abstract}

\section{Introduction}

Block cave has been accepted by the metalliferous community as the cheapest underground mining method of ore extraction for massive ore deposits which have relatively low strength compared to the host rock mass. This method has gained increased popularity in recent years, and many mines are currently thinking of adopting this method for deeper ore deposits. However, the benefit of this method is dependent on the progressive caveability of the roof. So improved understanding of the fracturing processes, the size distribution of the fragments, and the material flow behaviour are crucial to successful operations in caving processes. Furthermore, if deposits are associated with thick clay bands, modelling the flow of clay into the chutes is important as it may choke the chutes causing production delays.

With the rapid advancements in computer technology, numerical methods provide powerful tools for the analysis and design of engineering systems with complex factors that were either not possible or difficult using the conventional methods, often based on closed form analytical solutions (Brown and Bray, 1987). Analytical solutions are rarely found for rock mechanics problems that are of practical concern. This may be due to the difficulties in characterising the inhomogeneity of the rock mass under consideration, the structural discontinuities present in the rock mass, nonlinear constitutive behaviour of rocks, the boundary conditions, etc. These complexities associated with rock masses have necessitated the use of numerical methods.

The traditional mesh-based continuum methods, such as the finite element method (FEM), the finite difference method (FDM), and the boundary element method (BEM) have been employed for stress analysis 
and for simulating caving processes in mines. These methods often fail to simulate fracture and fragmentation and large material deformation or flow during a caving process accurately, because of mesh distortion resulting in spurious and numerically ill conditioned models. Recently, the extended finite element method (XFEM) has been applied to eliminate mesh distortion problems due to crack growth (Belytschko and Black, 1999; Moes et al., 1999, Belytschko et al., 2001). However, XFEM relies on a prescribed set of crack paths (Rabczuk et al., 2009), and is not well suited to modelling large numbers of interacting cracks present in arbitrary locations. Also, the simulation of large material flows and motion of fragments associated with caving present difficulties.

In this paper, the advantages of mesh-free method for solving large deformation and flow are highlighted over conventional mesh-based methods for simulating caving processes. A mesh-free method called SPH is used to demonstrate the applicability of this technique for simulating fracture and large scale material deformation observed in caving processes. Two different material behaviours (brittle and ductile) are considered to demonstrate caving processes associated with different rock material behaviours.

\section{SPH method}

SPH is a mesh-free method for the solution of partial differential equations. It was originally developed in 1977 by Gingold and Monaghan (1977) and independently by Lucy (1977), and has been traditionally used for the solution of complex fluid dynamics problems (Monaghan, 1992; Cleary et al., 2002, 2007; Imaeda and Inutsuka, 2002). In recent years, there has been a growing interest in applying SPH to a wide variety of solid mechanics problems (Libersky and Petschek, 1990; Wingate and Fisher, 1993; Gray et al., 2001; Gray and Monaghan, 2004; Liu et al., 2004; Cleary et al., 2006; Rabczuk et al., 2009). The problem geometry is discretised into 'particles' that represent specific material volumes. For details of SPH fundamentals, see Monaghan (1992, 2005) and Cleary et al. (2007).

The SPH method is based on the use of local interpolations on surrounding discrete particles to construct continuous field approximations. This is the basis of the spatial discretisation of governing equations. The interpolated value of a function $A$ at any position $\mathbf{r}$ can be expressed using SPH smoothing as proposed by Monaghan (1992):

$$
A(\mathbf{r})=\sum_{b} m_{b} \frac{A_{b}}{\rho_{b}} W\left(\mathbf{r}-\mathbf{r}_{b}, h\right)
$$

where $m_{b}$ and $\rho_{\mathrm{b}}$ are the mass and the density of particle $b$, and the sum is over all particles $b$ within a radius $2 h$ of $\mathbf{r}$. Here $W(\mathbf{r}, h)$ is a $C^{2}$ spline-based interpolation or smoothing kernel with radius $2 h$, that approximates the shape of a Gaussian function, but has compact support.

The gradient of the function $A$ is given by differentiating the interpolation Equation (1) to give:

$$
\nabla A(\mathbf{r})=\sum_{b} m_{b} \frac{A_{b}}{\rho_{b}} \nabla W\left(\mathbf{r}-\mathbf{r}_{b}, h\right)
$$

Using these interpolation formulae and suitable finite difference approximations for second order derivatives, one is able to convert parabolic partial differential equations into ordinary differential equations for the motion of the particles and the rates of change of their properties.

\subsection{SPH formulation for elastic-brittle solids}

The differential equations governing the structural responses and their SPH approximations are now presented.

\subsubsection{Continuity equation}

The continuity equation for an elastic solid is given as:

$$
\frac{d \rho}{d t}=-\rho \nabla \bullet \mathbf{v}
$$


where $\rho$ is the density and $\mathbf{v}$ is the velocity. The position vector from particle $b$ to particle $a$ is denoted by $\mathbf{r}_{a b}=\mathbf{r}_{a}-\mathbf{r}_{b}$, and $W_{a b}=W\left(\mathbf{r}_{a b}, h\right)$ is the interpolation kernel with smoothing length $h$ evaluated for the distance $\left|\mathbf{r}_{a b}\right|$. The SPH discretisation of the continuity equation from (Monaghan, 1992) is then:

$$
\frac{d \rho_{a}}{d t}=\sum_{b} m_{b}\left(\mathbf{v}_{a}-\mathbf{v}_{b}\right) \bullet \nabla W_{a b}
$$

where $\rho_{a}$ is the density of particle $a$ with velocity $\mathbf{v}_{\mathrm{a}}$, and $m_{b}$ is the mass of particle $b$ with velocity $\mathbf{v}_{b}$.

\subsubsection{Momentum equation}

The momentum equation used for elastic deformation of solids is:

$$
\frac{d \mathbf{v}}{d t}=\frac{1}{\rho} \nabla \bullet \boldsymbol{\sigma}+\mathbf{g}
$$

where $\boldsymbol{\sigma}$ is the stress tensor, and $\boldsymbol{g}$ denotes the body force.

The SPH discretisation of the momentum equation is:

$$
\frac{d \mathbf{v}_{a}}{d t}=\sum_{b} m_{b}\left(\frac{\boldsymbol{\sigma}_{a}}{\rho_{a}^{2}}+\frac{\boldsymbol{\sigma}_{b}}{\rho_{b}^{2}}+\Pi_{a b} \mathbf{I}\right) \bullet \nabla_{a} W_{a b}+\mathbf{g}_{a}
$$

where $\sigma_{\mathbf{a}}$ and $\sigma_{\mathbf{b}}$ are the stress tensors of particles $a$ and $b, \Pi_{a b}$ is a term (artificial viscosity) that produces a shear and bulk viscosity, and $\mathbf{g}_{\mathrm{a}}$ is the gravity at particle $a$.

Assuming Hooke's law with shear modulus $G$, the evolution equation for the deviatoric stress $\mathbf{S}(\mathbf{S}=\sigma+P \mathbf{I})$ is calculated using the Jaumann rate equation (Gray et al., 2001) as:

$$
\frac{d S^{i j}}{d t}=2 G\left(\dot{\varepsilon}^{i j}-\frac{1}{3} \delta^{i j} \dot{\varepsilon}^{i j}\right)+S^{i k} \Omega^{k j}+\Omega^{i k} S^{k j}
$$

where the component of the strain tensor $\dot{\boldsymbol{\varepsilon}}_{a}$ for particle $a$ is given in SPH form by:

$$
\dot{\varepsilon}_{a}^{i j}=\frac{1}{2} \sum_{b} \frac{m_{b}}{\rho_{b}}\left[\left(v_{b}^{i}-v_{a}^{i}\right) \frac{\partial W_{a b}}{\partial x_{a}^{j}}+\left(v_{b}^{j}-v_{a}^{j}\right) \frac{\partial W_{a b}}{\partial x_{a}^{i}}\right]
$$

The rotation tensor $\left(\Omega_{a}\right)$ that accounts for the large rotational effect is given as:

$$
\Omega_{a}^{i j}=\frac{1}{2} \sum_{b} \frac{m_{b}}{\rho_{b}}\left[\left(v_{b}^{i}-v_{a}^{i}\right) \frac{\partial W_{a b}}{\partial x_{a}^{j}}-\left(v_{b}^{j}-v_{a}^{j}\right) \frac{\partial W_{a b}}{\partial x_{a}^{i}}\right]
$$

\subsection{Advantages of SPH for modelling caving}

Caving involves large scale deformation and fracturing and a particle method offers several advantages over traditional mesh-based techniques (e.g. FEM and BEM). Firstly, nonlinear FE analysis for a fracturing structure can be computationally expensive and may pose stability problems when a large number of fractures are considered. The SPH method automatically takes account of the geometric nonlinearities of the problem.

The mesh-free nature of SPH makes it ideally suited for fracture processes that involve extreme deformations and a large number of discontinuities. SPH does not require any underlying grid structure to represent the geometry and so avoids the inaccuracies and instabilities associated with maintaining the integrity and quality of the mesh during large deformations. SPH is a Lagrangian method, in which the equations are solved on the particles that are fully advected with the material flow velocities. As a result, SPH is able to follow high speed structural motion without the need to include any explicit free surface tracking. 
One key feature of fracture simulation is the ability to model the disintegration of solids to produce fragments. One common approach is to explicitly simulate crack propagation with FEM by detaching elements along the sides of propagating cracks, which is accomplished by allowing the separation of the coincident (shared) nodes of the adjacent elements as the fracture surface evolves (Aliabadi and Rooke, 1991). This requires a very fine mesh to allow the propagation of damage and cracks to be accurately predicted and avoid mesh dependent fracture pathways. The averaged representation of damage in a continuum volume, implemented in a mesh-less method, means that the cracks are not constrained to propagate along specific element boundaries. This is even true for a coarse particle resolution model, allowing the natural development of fracture patterns. SPH has the ability to track new free (fracture) surfaces as they are generated during fracturing processes.

In general, fracturing is driven by the stress-strain history experienced by the material. Traditional Eulerian methods have difficulties in tracking the stress-strain history on a node by node basis and in predicting the evolution of damage in the specimen. The ability of each SPH particle to continuously represent the same volume of material provides a natural ability to track material history, and implement history dependent material properties, constitutive relationships and fracture criteria (Cleary et al., 2007). The history tracking ability of SPH thus provides a natural framework enabling the prediction of damage initiation and crack propagation. The dynamics of damage evolution is then able to be explicitly included in the analysis.

\section{Continuum damage law}

Dynamic fracturing is a complex phenomenon that involves the interaction between inherent or generated defects (flaws) and transient stress waves propagating within the material (Barker et al., 1978). The key issues in numerical modelling of damage are:

- representing inherent initial defects in a material

- characterising the level of fracture at a specific location in a material

- representing damage growth leading to fracture.

We use the SPH formulation for elastic solid deformation given in (Gray et al., 2001) and combine it with a continuum damage model to simulate brittle fracture. We used a modified form of the well-established Grady and Kipp damage model (Grady and Kipp, 1980). This model is widely used for the prediction of rock damage based on the local stress history and flaw distribution. To characterise the extent of fracture in a volume of material, a scalar damage parameter $D$ is used to indicate the volume averaged micro-fracture of the material represented by each SPH particle. The damage parameter inhibits the transmission of tensile stresses between particles and it evolves based on the strain history of each particle. The damage parameter lies between zero and unity. Materials with $D=0$ are undamaged or uncracked and are able to transmit the full tensile load, whereas materials with $D=1$ are fully damaged and cannot transmit any tensile load, thus creating a partial crack. Contiguous cracked material across a body leads to fragmentation.

Assuming a constant crack growth speed $C_{g}$, the damage $D(t)$ evolution is given by the integral equation:

$$
D(t)=\frac{4}{3} \pi C_{g}^{3} \int_{0}^{t} n^{\prime}(\varepsilon) \dot{\varepsilon}(1-D)(t-\tau)^{3} d \tau
$$

where $\varepsilon$ is the tensile strain, $n^{\prime}$ is the change in number of flaws, $t$ is the current time, $\tau$ is the past times for history integration.

An approximate differential form of Equation (10) (from Grady and Kipp, 1980) is:

$$
\frac{d D^{1 / 3}}{d t}=\frac{(m+3)}{3} \alpha^{1 / 3} \varepsilon^{m / 3}
$$

where $\alpha$ is a material constant calculated from three material fracture parameters $k, m$, and $C_{g}$ :

$$
\alpha=\frac{8 \pi C_{g}^{3} k}{(m+1)(m+2)(m+3)}
$$


The original Grady-Kipp model is valid only for one-dimensional plain strain problems. For use in two or three-dimensions, the full stress-strain field needs to be reduced to the one-dimensional analysis framework outlined in Grady and Kipp (1980). One proposed method replaces the longitudinal strain by the volumetric strain (average of the three longitudinal strain components) (Thorne et al., 1990). This poses difficulties when some of the longitudinal strain components are compressive and others are tensile, and their magnitudes are such that the resulting volume strain is compressive. In this case, no damage evolution will occur under a compressive strain according to this damage model, but in reality damage accumulation should take place due to the tensile component(s) of the strain tensor. To avoid this problem, Melosh et al. (1992) introduced an effective tensile strain given by:

$$
\varepsilon_{\text {eff }}=\sigma_{\max } /\left(K+\frac{4}{3} G\right)
$$

where $\sigma_{\max }$ is the maximum positive principal stress, and $K$ and $G$ are the bulk modulus and the shear modulus of the material.

Damage then accumulates when the effective strain exceeds a threshold value $\varepsilon_{\min }$ :

$$
\varepsilon_{\text {min }}=(V k)^{-1 / m}
$$

where $V$ is the volume of each SPH particle.

This effective strain is used in the evolution Equation (11) to predict the damage state of the material. So for damage evolution in SPH we use the combination of the differential version of the Grady-Kipp model (Equation (11)) and the effective tensile strain (Equation (13)). The total stress tensor is rotated into its principal coordinate frame where the tensile stress is easiest to identify and then to scale only the tensile components of the principal stresses. These are rotated back to the global coordinate frame to give the post-damage stresses used in the stress evolution and momentum equations as in Gray et al. (2001).

\section{$4 \quad$ Model geometry}

The main objective of this study was to demonstrate the application of the SPH method for simulating caving processes. The geometry of the model is shown in Figure 1 along with dimensions. An undercut with $4.5 \mathrm{~m}$ height, $250 \mathrm{~m}$ wide and $800 \mathrm{~m}$ long is to be created. These represent typical dimensions for longwall mining in stratified rock mass. The undercut (Figure 1) is at a depth of $316 \mathrm{~m}$ below the surface. The rock mass geometry considered for this problem is $3 \mathrm{~km}$ long (along the $\mathrm{X}$-axis), $2 \mathrm{~km}$ wide (along the $\mathrm{Y}$-axis) and $0.5 \mathrm{~km}$ deep (along the Z-axis). Here the three-dimensional geometry was simplified to a two-dimensional plane strain numerical model. The SPH particles (interpolation points) were uniformly distributed over the model geometry. The excavation is simulated by gradually removing particles in the undercut gallery.

Simplified stratigraphic units are assumed for the numerical models. The stratified layer thicknesses and their material properties are given in Table 1. The yield strength in Table 1 is assumed to be $75 \%$ of the unconfined compressive strength (UCS) of the material and the hardening modulus is assumed to be $20 \%$ of the Young's modulus. For boundary conditions, the free surface was not constrained and the bottom surface was fixed in all the directions. Roller boundary conditions were prescribed on all sides to allow movement of the material only in the vertical direction.

Gravity loading was applied and an average overburden density of $2,320 \mathrm{~kg} / \mathrm{m}^{3}$ was assumed in the models. The undercut was initiated from one end and gradually progressed to the other end. The model geometry selected for the initial caving models was designed to provide a reasonably detailed understanding of the caving behaviour. No attempt was made to introduce strain softening, as the softening parameters which are a function of plastic strain were unknown. The strata interface properties were defined in terms of damage values randomly varying from 0 to 1 along the interface. A damage value of unity indicates a completely disjointed interface, whilst a damage value of zero indicates an unfractured or intact interface. 


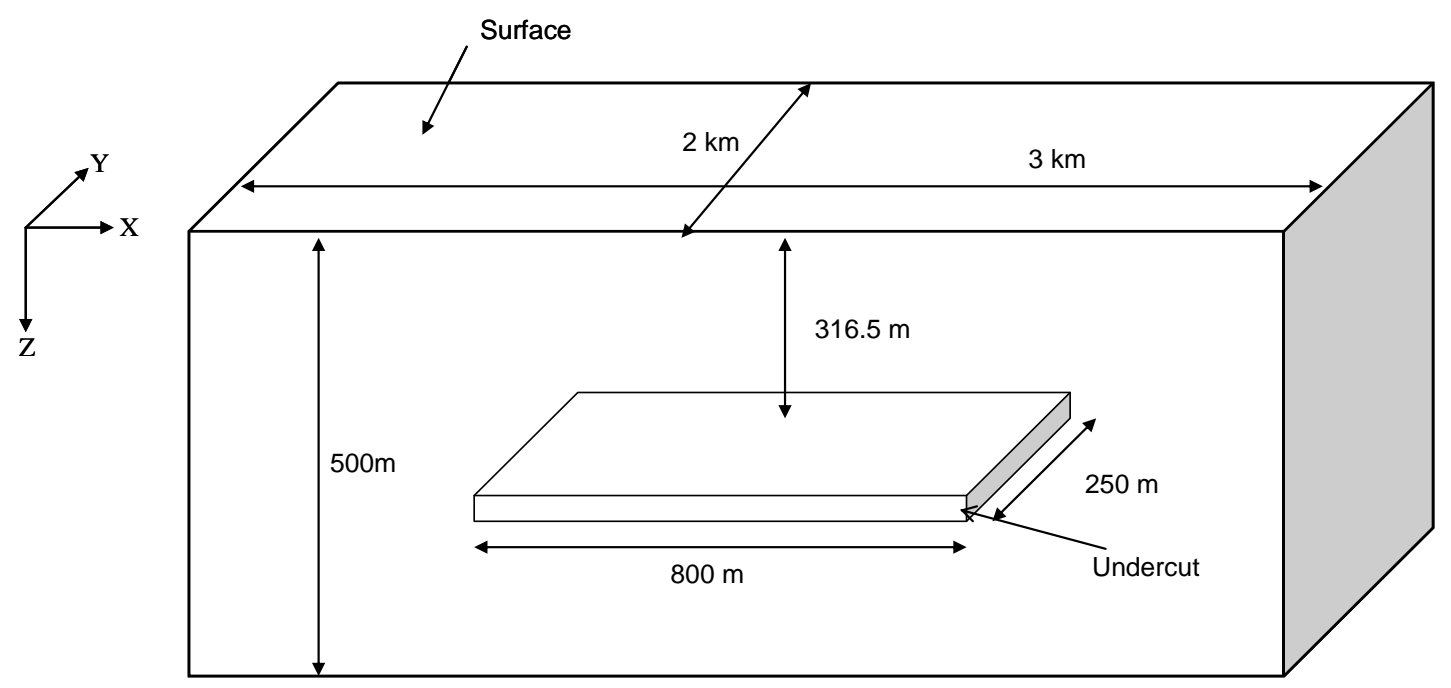

Figure 1 A 3D block indicating the undercut geometry

Table 1 Strata sequence with rock unit thickness and depth below surface

\begin{tabular}{lllllll}
\hline $\begin{array}{l}\text { Strata } \\
\text { Layers }\end{array}$ & $\begin{array}{l}\text { Depth Below } \\
\text { Surface } \\
(\mathbf{m})\end{array}$ & $\begin{array}{l}\text { Thickness } \\
(\mathbf{m})\end{array}$ & $\begin{array}{l}\text { Young's } \\
\text { Modulus } \\
\mathbf{E}(\mathbf{G P a})\end{array}$ & $\begin{array}{l}\text { Poisson's } \\
\text { Ratio } \\
(\mathbf{v})\end{array}$ & $\begin{array}{l}\text { UCS } \\
(\mathbf{M P a})\end{array}$ & $\begin{array}{l}\text { Yield Strength } \\
\text { for Model-II } \\
(\mathbf{M P a})\end{array}$ \\
\hline Surface & 0.0 & & & & & \\
Layer-1 & 187.8 & 187.8 & 4.6 & 0.2 & 5.03 & 3.8 \\
Layer-2 & 213.8 & 26.1 & 6.07 & 0.19 & 6.14 & 4.6 \\
Layer-3 & 218.5 & 4.7 & 3.00 & 0.25 & 5.32 & 4.0 \\
Layer-4 & 239.3 & 20.7 & 7.44 & 0.2 & 9.83 & 7.4 \\
Layer-5 & 244.0 & 4.7 & 3.39 & 0.2 & 3.90 & 2.9 \\
Layer-6 & 257.6 & 13.6 & 6.43 & 0.15 & 6.60 & 5.0 \\
Layer-7 & 283.2 & 25.7 & 6.66 & 0.18 & 3.91 & 2.9 \\
Layer-8 & 286.2 & 3.0 & 3.00 & 0.25 & 4.07 & 3.1 \\
Layer-9 & 305.0 & 18.8 & 5.7 & 0.2 & 2.89 & 2.2 \\
Layer-10 & 309.4 & 4.4 & 5.7 & 0.2 & 2.89 & 2.2 \\
Layer-11 & 316.5 & 7.1 & 3.00 & 0.25 & 6.00 & 4.5 \\
Layer-12 & 321.0 & 4.5 & 3.00 & 0.25 & 6.00 & 4.5 \\
Layer-13 & 324.6 & 3.7 & 7.97 & 0.24 & 8.36 & 6.3 \\
Layer-14 & 327.6 & 3.0 & 3.00 & 0.25 & 3.69 & 2.8 \\
Layer-15 & 362.6 & 35.0 & 8.48 & 0.2 & 7.74 & 5.8 \\
Base & 500.0 & 137.4 & 5.48 & 0.2 & 20.01 & 15.0 \\
\hline
\end{tabular}

\section{$5 \quad$ Simulation results and discussions}

We considered two different approaches of numerical simulations. In the first case (case I), the entire problem domain was modelled with SPH particles, and in the second case (case II), the coupled SPH-Finite Element Method (FEM) was used. In the coupled (SPH-FEM) simulation, the part of the domain surrounding 
the excavation is modelled with SPH particles, and the remaining regions were modelled with finite elements to reduce the computation run time.

\subsection{Case I: SPH simulation}

Two different models were constructed for the same geometry for this case as given below:

- Model-I: material model with a large yield/failure strength to capture brittle fracturing in the caving process. This rock material behaviour is predominantly elastic-brittle.

- Model-II: material model with a lower yield/failure strength compared to Model I so that the rock mass behaves in ductile fashion.

In all these models, the height of the undercut was assumed to be $4.5 \mathrm{~m}$ and the undercut gallery was excavated incrementally from one end.

\subsubsection{Model-I: Brittle fracturing}

The gravitational stresses were first equilibrated before commencement of the excavation. Figure 2(A) shows the distribution of the effective overburden stress due to gravity loading from the surface to a depth of $500 \mathrm{~m}$ (with the stress scale shown at the bottom of each sub-figure). An undercut of height $h$ and width equal to $2.66 h$ is made in Figure 2(B). Due to the excavation, the stresses redistribute around the excavation resulting in a destressed zone above the excavation and increased stress concentrations on either side of the excavation. As the excavation is gradually progressed towards left by removing material, both the extent of the destressed zone and the stress concentrations are increased (Figure 2(C)). With further increase in the excavation distance, the rock material starts to move down into the excavated void, whilst the layers above start to delaminate as observed in Figure 2(D).

With further increase in the excavation span (Figure 2(E)), the layer immediately above the void begins to fall apart with high stress concentration generated around the excavation front. An important feature to note is the bridging of the layer above the caved material. As the excavation front further progresses, the destressed zone widens and further delamination of the layers occurs with the layer immediately above the caved material still bridging the load (Figure 2(F)). In Figure 2(G), continued deformation of more layers accompanied by further widening of the destressed zone and increased stress concentrations are observed. The layer that bridged has now been deformed with a tensile fracture emanating at the lower surface somewhere about its mid span. Further, we observe the separation of layers higher up.

In Figure 2(H), fracturing and unravelling of the layers/beams (left part of the delaminated top layer) occur at a span of about 4.5 times the height of excavation. This span-height ratio depends on the bending resistance, layer strength, and overburden stress. When the top (roof) layer touches the lower (floor) layer, it transfers load through the points of contact. The load is transferred to the edges of the undercut in an arch fashion, increasing the stress concentration at the excavation front, as seen in Figure 2(I). As the roof layer contacts the floor of the excavation, the stress concentrations continue to develop at the points of contact (between the roof and the floor). In addition, as the excavation front progresses (Figure 2(J)), cracking and collapse of the top layer occurs towards the static front to the right. High stresses are observed at intermittent distances (contact points) (around 3.5 times the height of the excavation) along the floor (Figure 2(K)).

With further progression of the excavation front, localised arches or bridges (greenish colour in Figure 2(L)) progressively build up while the layer at the top continues to delaminate. The separated layers continue to break up at a distance of around four to five times the height of the excavation. This is accompanied by increased stress concentrations surrounding the destressed zone.

Figure 2(M) shows that the delamination continues as the excavation progresses, and the destressed zone above the delaminated layer increases. This is because the delamination acts as a free surface preventing transfer of tensile stresses. High stress concentrations occur around the corners of the destressed zone. When the delamination span reaches a critical length, a crack initiates at the centre of the interface between destressed and stressed zones. This crack propagates and causes failure of a large intact rock that falls on top of the debris below due to gravity, as shown in Figure 2(N). This type of failure can cause an airblast, similar to that happened in Northparkes Mine in Australia (Bailey, 2003). 

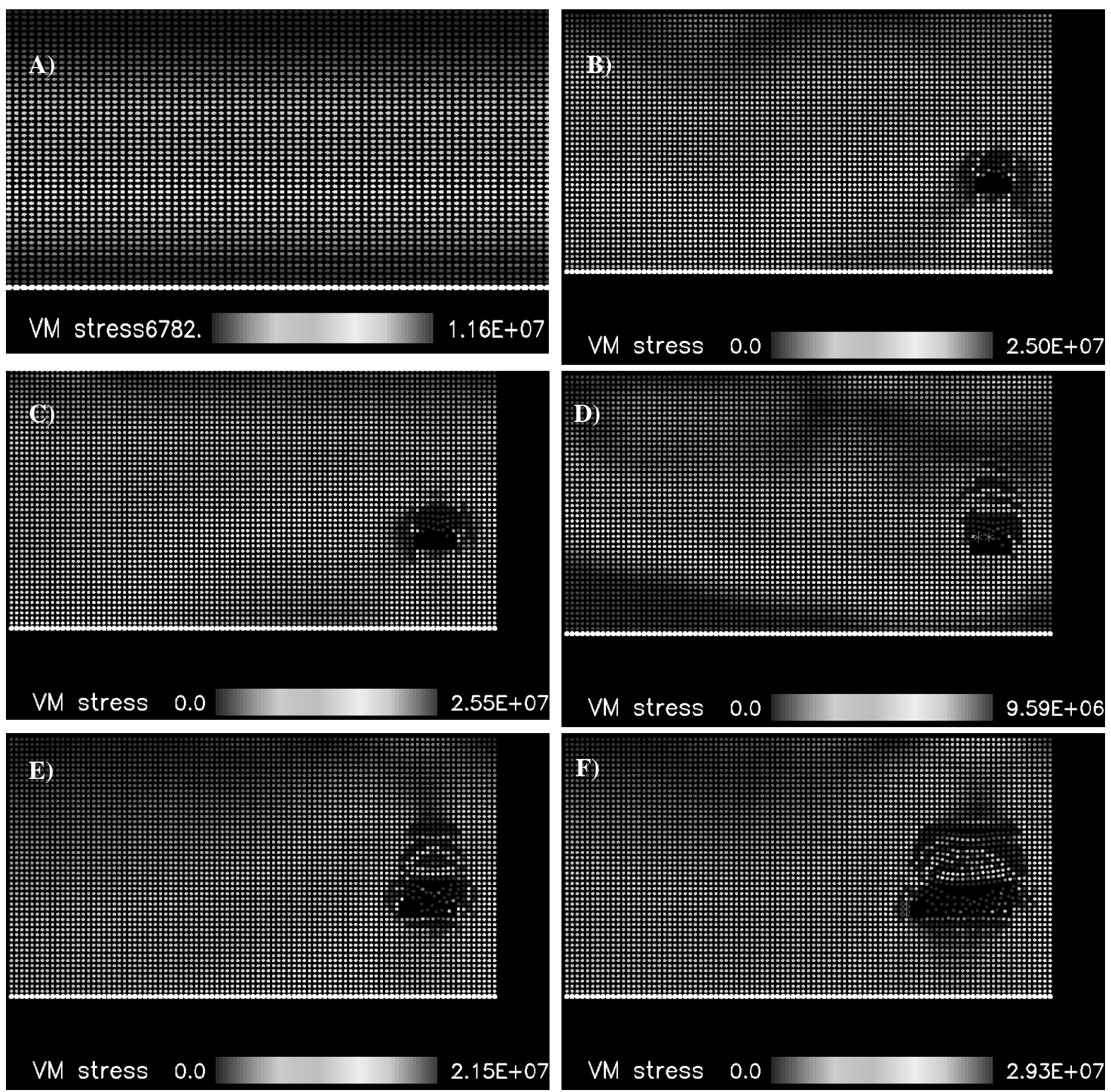

Fi)

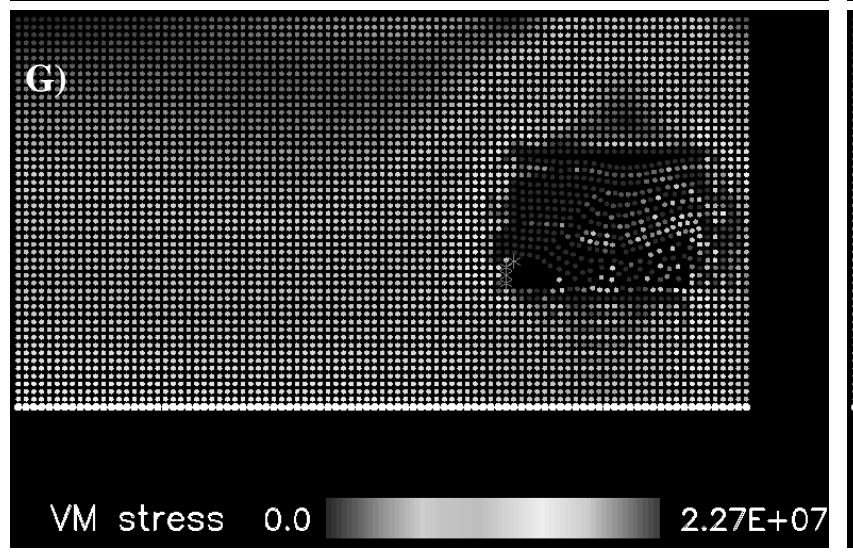

VM stress 0.0

$2.93 E+07$

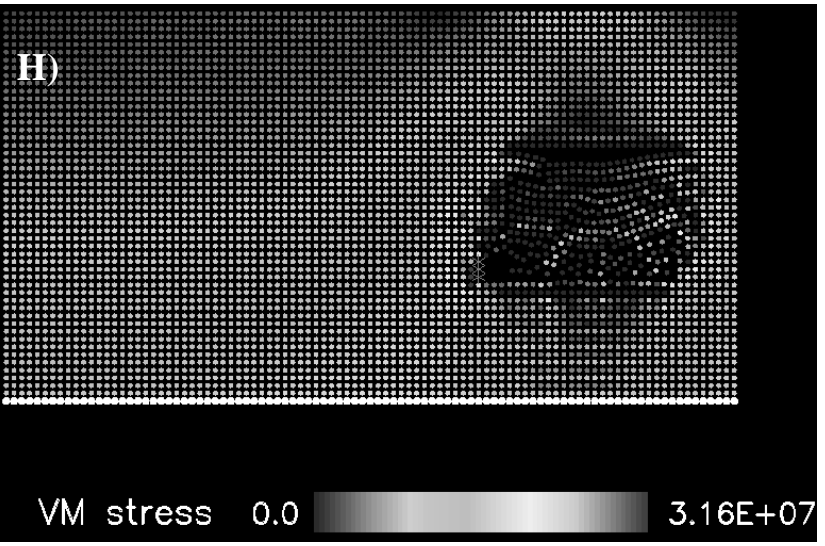

Figure 2 continued . 

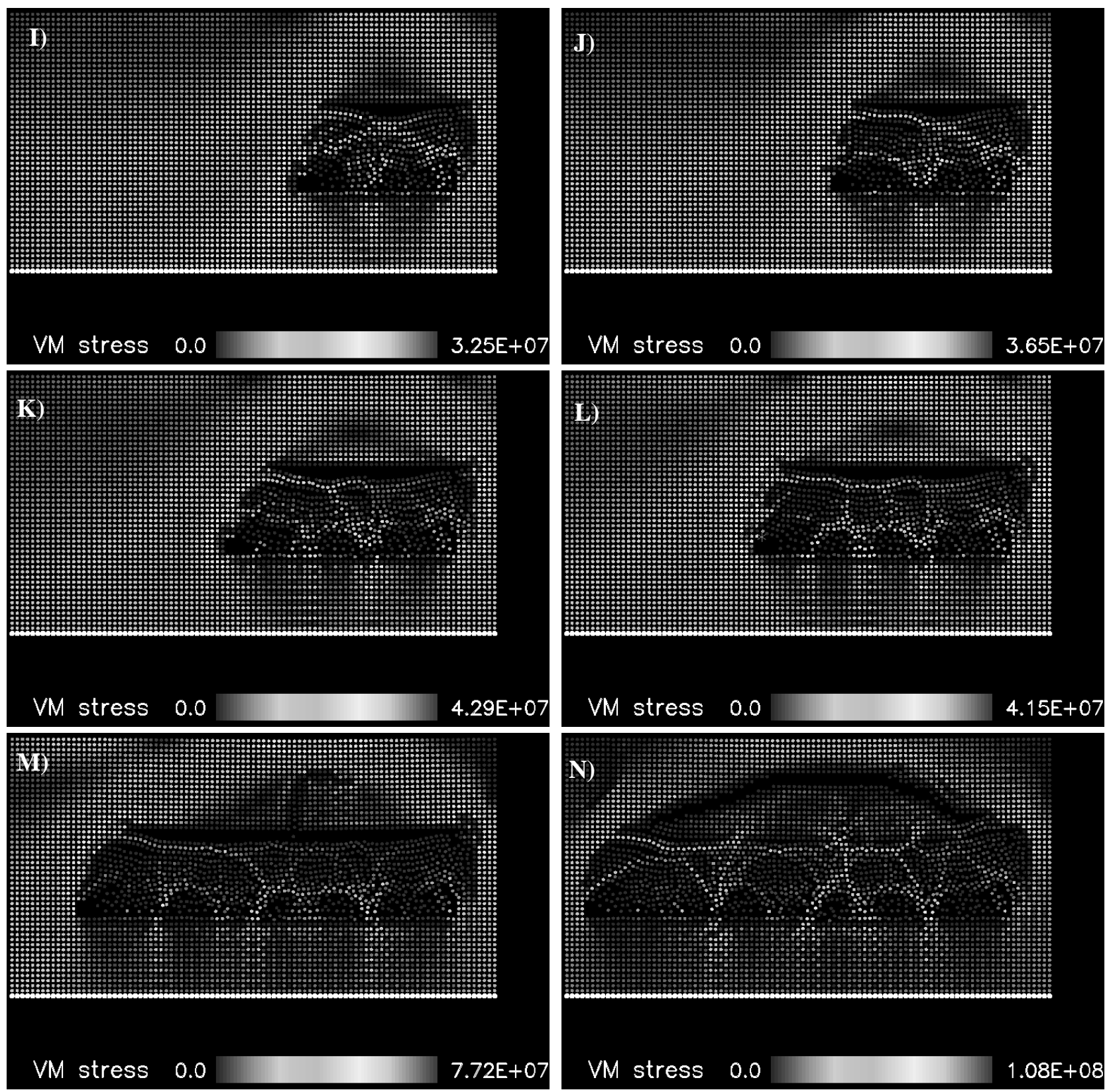

Figure 2 Case I: Redistribution of effective stress and delamination and fracturing of layers at various stages of caving in an elastic-brittle rock mass (effective stress scale is shown at the bottom of each sub-figure)

\subsubsection{Model-II: Elasto-plastic deformation}

Next we consider an elasto-plastic model for the rock mass. In this case, the yield strengths were chosen to be about $75 \%$ of the UCS of the individual layers. The hardening modulus was chosen to be $20 \%$ of the Young's modulus for each layer.

In the case of rock mass following elasto-plastic material behaviour, majority of the bulk movement of the material occurs by plastic flow. This large material deformation increases the plastic strain up to 50\% in this case, as shown in Figure 3. Unlike the elastic-brittle fracture model, distinct crack initiation and propagation during the plastic deformation is not observed. The caving process is primarily dominated by the plastic flow of material, and high plastic strains can be identified at the contact zones between the roof and the floor. In practice, majority of rocks behave in quasti-brittle manner, and realistic rock behaviour would be bounded somewhere between these two extreme (elastic-brittle and elasto-plastic) material models. 

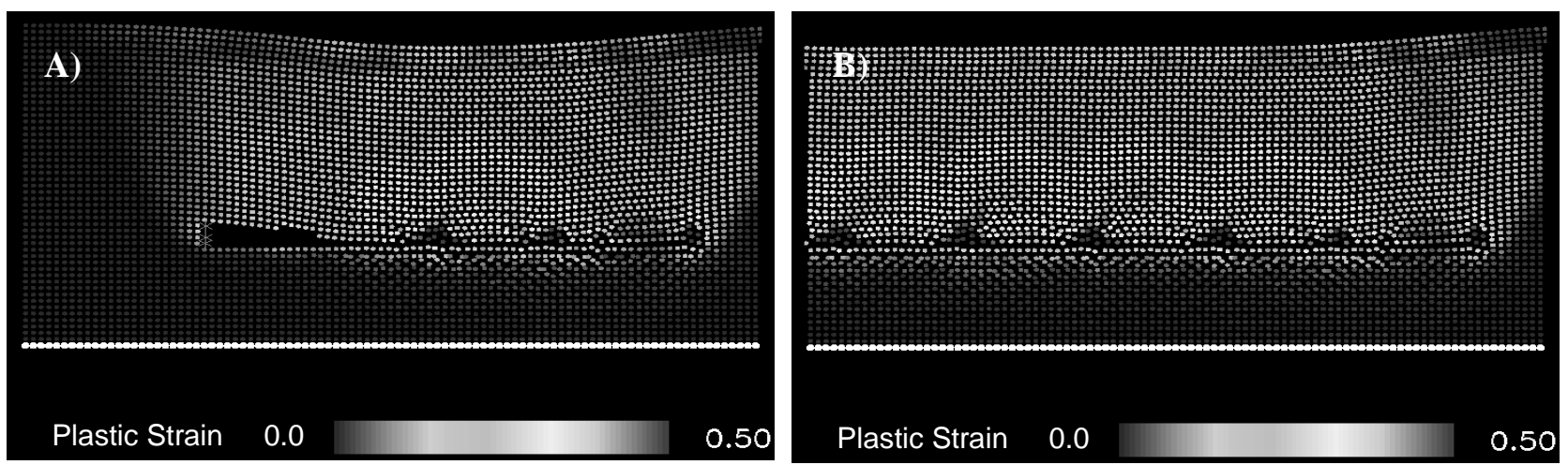

Figure 3 Case I: Plastic flow of rock mass at two different stages in elasto-plastic material model-II

\subsection{Case II: Coupled SPH-FEM simulation}

Lastly, the application of coupled solvers for caving process simulation is presented. In this example, coupled SPH-FEM method was used to simulate the caving process. The model geometry is shown in Figure 4(A). The numerical model was constructed by combining a SPH domain with a FE domain. The immediate two layers above the undercut gallery were modelled with SPH particles, and the remaining zones further up were modelled with finite elements (Figure 4(A)). An elasto-plastic analysis was used in this simulation. For this problem, the yield strength values were assumed to be low; $300 \mathrm{kPa}$ for the FE domain, $100 \mathrm{kPa}$ for the layer immediately above the undercut, and $300 \mathrm{kPa}$ for the middle layer. The immediate two layers above the undercut gallery were modelled with SPH particles, and the layers further up were modelled with finite elements. The advantage of the coupled approach is that it reduces the computational time required for the simulation.

When the first cut is made (Figure 4(B)), the roof starts to deform into the void, and fractures initiate at the corners and gradually extend upwards to form a zone of bulk fracturing - called the cave development zone, which is about twice the height of the undercut. During this process, the layer immediately above the void separates from the top layer. The material deformation is found to extend in an elliptic shape up to approximately 10 times the height of the excavation, as seen by lighter plastic zones (Figure 4(B)). With further deformation, the layers begin to fragment, forming a well-developed caved zone (indicated in darker spotted areas in Figures 4(C) and (D)). When the second cut is undertaken, the roof of the second cut starts to deform and caves into the existing void (Figures 4(E) and 4(F)). A well-developed elliptic shape of material movement can be observed, which is further widened and extended upwards. This caving shape and the associated deformation process resemble material movement generally observed in the field. 

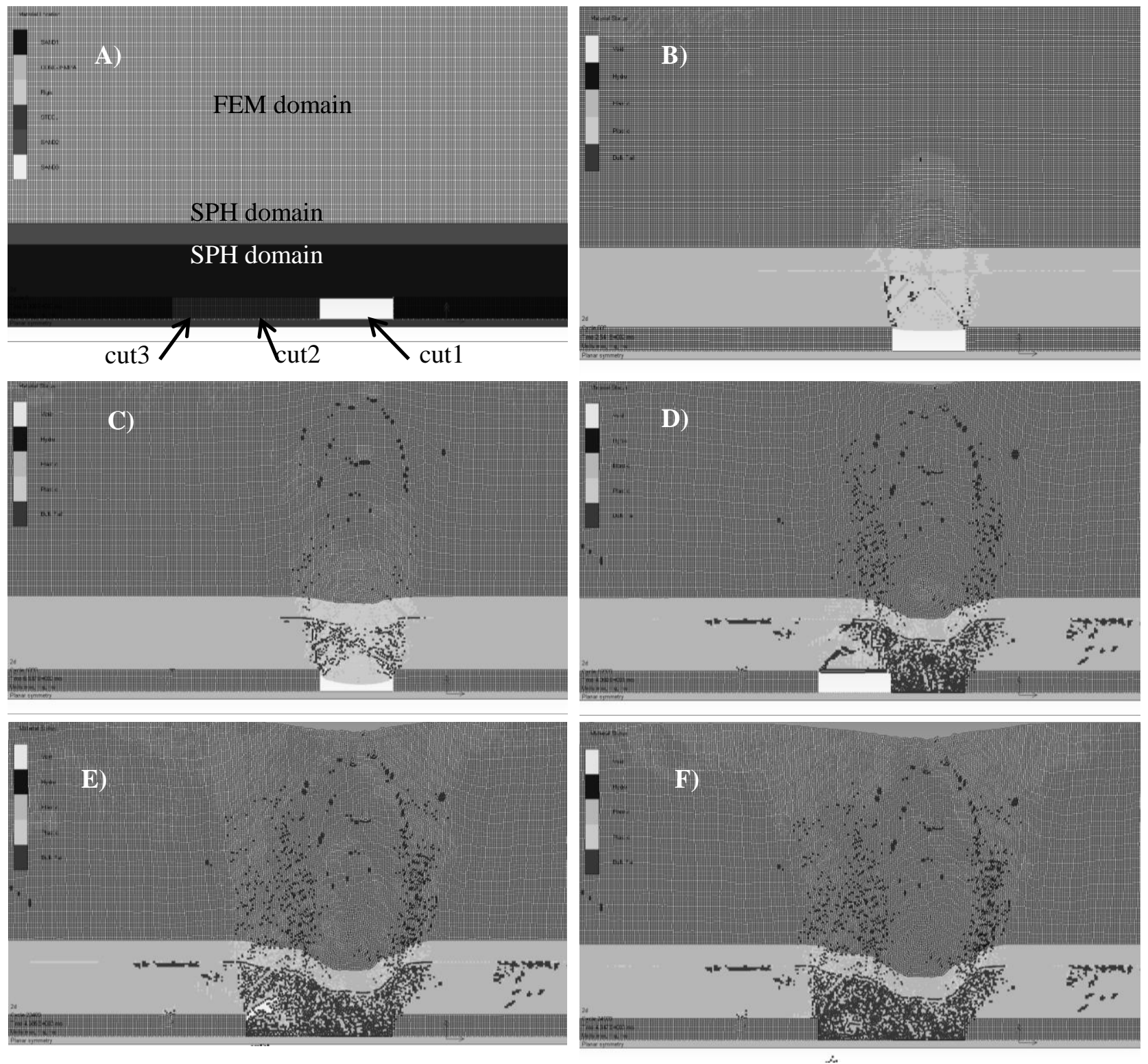

Figure 4 Case II: Elliptical shape cave development and material flow as modelled using SPH combined with FEM; (a) geometry of the model; (b) to (f) cave development and bulk material flow)

\section{Conclusion}

In this paper, we have demonstrated the application of a mesh-free method, called Smoothed Particle Hydrodynamics (SPH), for modelling a caving process. Rock caving is a large deformation problem where fracture formation and fragmentation are crucial. This makes the application of a mesh-free method advantageous. It has been shown that SPH can model the complex physical processes associated with caving, which are usually difficult to simulate using traditional mesh-based methods.

It has been shown that SPH can predict the mechanistic aspects of caving processes. In particular, the material deformation, fracture formation and fragmentation that lead to progressive roof collapse are well captured. Two different material behaviours for the rock mass were considered, namely, elastic-brittle and elasto-plastic. In the case of elastic-brittle rocks, the caving process is predominantly governed by cracking, delamination, and fracturing across rock layers. For the elasto-plastic rocks, the failure is not governed by distinct fracture planes. Rather, the caving process is predominantly dominated by plastic flow with large deformations. The SPH simulation results appear to capture the mechanisms of fracture formation and fragmentation during caving for both of these extreme cases of rock material behaviours. It was also found 
that the cave development evolves in an elliptic shape, similar to that observed in the field. Understanding the key mechanisms of fracture processes and the resulting fragmentation provide new insights for improved design of various aspects of caving, such as panel design, draw point spacing, draw control, etc.

The numerical simulation results presented here demonstrate that the SPH based mesh-free computational framework can be an effective numerical modelling tool and is well suited to simulating large deformation and flow problems accompanied by fracture formation and fragmentation. Many of the mesh-free methods require a higher computational time compared to that of the conventional mesh-based methods. However, for rock caving problems which involve fracturing and fragmentation, SPH potentially offers significant advantages that may offset the computational time limitation. Moreover, several approaches could be adopted to increase the computational efficiency of SPH, such as variable resolution SPH, parallel SPH algorithms and coupling of mesh-free and mesh-based methods (e.g. SPH-FEM). The last approach has been demonstrated via an example of coupled SPH-FEM simulation of rock caving, which utilises the benefits of both mesh-free and mesh-based methods. For example, in the problem considered, the use of the coupled method was found to be more computationally efficient, because it allowed the use of a course finite element mesh further away from the fracturing and caving regions, thus reducing the overall computer time for simulations.

\section{Acknowledgements}

The financial support under CSIRO Capability Development funds is gratefully acknowledged. The support provided by Dr. Srini Bandla of Leap Australia Pty Ltd is thankfully acknowledged.

\section{References}

Aliabadi, M.H. and Rooke, D.P. (1991) Numerical Fracture Mechanics, Computational Mechanics Publications and Kluwer Academic Publishers.

Bailey, J. (2003) Findings and recommendations: Inquest into the deaths of R. Bodkin; M. House; S. Osman and C. Lloyd-Jones on the 24th November, 1999 at the E26 Lift 1 Mine, Northparkes Mines, Parkes, New South Wales, NSW Coroners Court.

Barker, D.B., Fourney, W.L. and Dally, J.W. (1978) The influence of stress waves on explosive induced fragmentationborehole crack network, 19th US Symposium on Rock Mechanics, Lake Tahoe.

Belytschko, T. and Black, T. (1999) Elastic crack growth in finite elements with minimal remeshing, International Journal for Numerical Methods in Engineering, Vol. 45, pp. 601-620.

Belytschko, T., Moes, N., Usui, S. and Parimi, C. (2001) Arbitrary discontinuities in finite elements, International Journal for Numerical Methods in Engineering, Vol. 50, pp. 993-1013.

Brown, E.T. and Bray, J. (1987) Analytical and Computational Methods in Engineering Rock Mechanics, Allen \& Unwin.

Cleary, P., Ha, J., Alguine, V. and Nguyen, T. (2002) Flow modelling in casting processes, Applied Mathematical Modelling, Vol. 26, pp. 171-190.

Cleary, P.W., Prakash, M. and Ha, J. (2006) Novel applications of smoothed particle hydrodynamics (SPH) in metal forming, Journal of Materials Processing Technology, Vol. 177, pp. 41-48.

Cleary, P.W., Prakash, M., Ha, J., Stokes, N. and Scott, C. (2007) Smooth particle hydrodynamics: status and future potential, Progress in Computational Fluid Dynamics, Vol. 7, pp. 70-90.

Gingold, R.A. and Monaghan, J.J. (1977) Smoothed particle hydrodynamics - Theory and application to non-spherical stars, MNRAS, Vol. 181, pp. 375-389.

Grady, D.E. and Kipp, M.E. (1980) Continuum modelling of explosive fracture in oil shale, International Journal of Rock Mechanics and Mining Sciences and Geomechanics Abstracts, Vol. 17, pp. 147-157.

Gray, J.P. and Monaghan, J.J. (2004) Numerical modelling of stress fields and fracture around magma chambers, Journal of Volcanology and Geothermal Research, Vol. 135, pp. 259-283.

Gray, J.P., Monaghan, J.J. and Swift, R.P. (2001) SPH elastic dynamics. Computer Methods in Applied Mechanics and Engineering, Vol. 190, pp. 6641-6662.

Imaeda, Y. and Inutsuka, S-I. (2002) Shear Flows in Smoothed Particle Hydrodynamics, The Astrophysical Journal, Vol. 569, pp. 501-518.

Libersky, L.D. and Petschek, A.G. (1990) Smooth particle hydrodynamics with strength of materials, In Advances in the Free-Lagrange Method, Trease and Crowley (eds), Berlin: Springer.

Liu, Z.S., Swaddiwudhipong, S. and Koh, C.G. (2004) High velocity impact dynamic response of structures using SPH method, International Journal of Computational Engineering Science, Vol. 5, pp. 315-326. 
Lucy, L.B. (1977) A numerical approach to the testing of the fission hypothesis, Astronomical Journal, Vol. 82, pp. 1013-1024.

Melosh, H.J., Ryan, E.V. and Asphaug, E. (1992) Dynamic fragmentation in impacts: hydrocode simulation of laboratory impacts, Journal of Geophysical Research, Vol. 97, pp. 14735-14759.

Moes, N., Dolbow, J. and Belytschko, T. (1999) A finite element method for crack growth without remeshing, International Journal for Numerical Methods in Engineering, Vol. 46, pp. 131-150.

Monaghan, J.J. (1992) Smoothed particle hydrodynamics, Annual Review of Astronomy and Astrophysics, Vol. 30, pp. 543-574.

Monaghan, J.J. (2005) Smoothed particle hydrodynamics, Reports on Progress in Physics, Vol. 68, pp. $1703-1759$.

Rabczuk, T., Song, J.H. and Belytschko, T. (2009) Simulations of instability in dynamic fracture by the cracking particles method, Engineering Fracture Mechanics, Vol. 76, pp. 730-741.

Thorne, B.J., Hommert, P.J. and Brown, B. (1990) Experimental and computational investigation of the fundamental mechanisms of cratering, 3rd International Symposium on Rock Fragmentation by Blasting, Brisbane, Australia, pp. $117-124$

Wingate, C.A. and Fisher, H.N. (1993) Strength Modeling in SPHC, Los Alamos National Laboratory, Report No. LAUR-93-3942. 
